\section{Fatores de risco cardiovascular e fatores associados em escolares do Município de Belém, Pará, Brasil}

\author{
Cardiovascular risk and associated factors in \\ schoolchildren in Belém, Pará State, Brazil
}

\section{Factores de riesgo cardiovascular y los factores asociados en escolares en Belém, Pará, Brasil}

Simone Augusta Ribas 1,2

Luiz Carlos Santana da Silva ${ }^{2}$

\author{
${ }^{1}$ Universidade do Estado do \\ Rio de Janeiro, Rio de Janeiro, \\ Brasil. \\ 2 Universidade Federal do \\ Pará, Belém, Brasil. \\ Correspondência \\ S. A. Ribas \\ Universidade do Estado do \\ Rio de Janeiro. \\ Rua Alexandre Ramos 535 \\ apto. 502, Rio de Janeiro, $R$ \\ 22735-140, Brasil. \\ ribasnut@yahoo.com.br
}

\begin{abstract}
This cross-sectional study aimed to identify risk factors for cardiovascular disease in a stratified cluster sample of 557 schoolchildren (6-19 years) in Belém, Pará State, Brazil. Potential risk factors were obesity, hypertension, dyslipidemia diabetes, smoking, physical inactivity, and atherogenic diet. Socio-demographic and lifestyle variables were tested in a binary logistic regression model. The most prevalent risk factors were overweight (20.4\%), dyslipidemia (48.1\%), and physical inactivity (66.2\%). Children below ten years of age and those from higher-income families and with higher maternal schooling showed greater odds of developing overweight; mean while, those with overweight were more prone to developing hypercholesterolemia and hypertriglyceridemia. The findings point to the need to implement strategies to prevent overweight in early childhood, through balanced nutrition and regular physical activity, in order to effectively reduce the prevalence of risk factors in schoolchildren.
\end{abstract}

Students; Cardiovascular Diseases; Risk Factors

\section{Resumo}

Este estudo transversal visou a identificar fatores de risco para doença cardiovascular em uma amostra, estratificada por conglomerados, de 557 escolares (6-19 anos) de Belém, Pará, Brasil. Os fatores de risco investigados foram obesidade, hipertensão arterial, dislipidemia, diabetes, tabagismo, sedentarismo e dieta aterogênica. Variáveis sociodemográficas e relacionadas ao estilo de vida foram testadas no modelo de regressão binária logística. Os fatores de risco prevalentes foram excesso de peso (20,4\%), dislipidemia (48,1\%) e sedentarismo (66,2\%). Constatou-se que os escolares abaixo de dez anos e os provenientes das famílias de maior renda e com maior escolaridade materna apresentaram mais chances de desenvolverem excesso de peso; por sua vez, os escolares com excesso de peso foram os mais propensos a desenvolver hipercolesterolemia e hipertrigliceredemia. Diante desse quadro, faz-se necessária, ainda na primeira infância, a implantação de estratégias para controle de excesso de peso, por meio da alimentação balanceada e da prática física regular, para que se possa reduzir de forma efetiva a prevalência de fatores de risco em escolares nesta cidade.

Estudantes; Doenças Cardiovasculares; Fatores de Risco 


\section{Introdução}

Dados epidemiológicos do Ministério da Saúde do Brasil para a cidade de Belém, Pará, revelaram que a mortalidade por doenças cardiovasculares constitui-se na principal causa de morte (50\%). Embora tais resultados revelem um percentual preocupante, percebe-se que são raros os estudos nacionais sobre prevalência de fatores de risco ateroscleróticos incluindo crianças e/ou adolescentes nessa região. A prevalência de dislipidemia para a cidade de Belém é desconhecida, o que dificulta o estabelecimento, a médio e longo prazos, de estratégias de intervenção que possam mudar o perfil de mortalidade associado a doenças cardiovasculares observadas neste município ${ }^{1}$.

As doenças cardiovasculares fazem parte do grupo das doenças crônicas não transmissíveis, as quais compõem a síndrome plurimetabólica (obesidade, hipertensão, diabetes mellitus e dislipidemia), causada por fatores de risco resultantes de mudanças de hábitos de vida 2 . Evidências científicas demonstraram, também em populações pediátricas, a associação positiva entre excesso de peso e dislipidemia ${ }^{3,4}$, bem como outros fatores de risco: hipertensão arterial, resistência à insulina e diabetes mellitus tipo 25,6 , ressaltando a importância do diagnóstico precoce.

Nesse contexto, o espaço escolar tornou-se local privilegiado, factível e oportuno para a obtenção de informações representativas sobre a saúde e nutrição 7 , pouco disponíveis no que se refere a crianças maiores de cinco anos e adolescentes da Região Norte.

Desse modo, o presente trabalho teve o objetivo de investigar a prevalência dos principais fatores de risco para doenças cardiovasculares e identificar fatores associados a eles em escolares da cidade de Belém.

\section{Métodos}

Estudo de delineamento transversal realizado em uma amostra representativa de escolares, entre 6 e 19 anos, matriculados em instituições de ensino fundamental e médio da rede pública e privada do Município de Belém, no período de outubro a novembro de 2005. Nesse ano, a população escolar era constituída por 313.866 estudantes de 293 escolas de ensino fundamental e médio (215 públicas e 78 particulares), de acordo com o censo escolar de 2004 fornecido pela Secretaria de Estado de Educação (SEDUC) do Pará 8 .

Para determinar o tamanho da amostra, foi estimado o erro de $3 \%$ desta e a prevalência de dislipidemia de $13 \%$ para cidade de Belém 9, utilizando-se o intervalo de 95\% de confiança. O tamanho da amostra inicial constituiu-se de 482 indivíduos e, prevendo-se perda de até $20 \%$, estimou-se em 580 estudantes para a amostra final.

A amostra foi selecionada por conglomerado em dois estágios. No primeiro, 29 escolas (21 públicas e 8 particulares) foram escolhidas aleatoriamente a partir da listagem fornecida pela SEDUC, de forma que representasse cada região administrativa da área metropolitana do município. No segundo estágio, das escolas sorteadas, 58 turmas foram selecionadas de forma aleatória, mas que atendessem, no mínimo, 30 alunos de cada sexo em cada grupo etário (6-9, 10-12, 13-15 e 16-19 anos). Participantes com pré-existência de doenças crônicas ou genéticas, assim como gestantes ou indivíduos utilizando hipolipemiantes, foram excluídos do estudo.

Todas essas informações foram obtidas mediante questionário fornecido previamente à instituição sorteada e distribuída aos pais ou responsáveis que participaram deste estudo. Por meio do instrumento, foram obtidas, ainda, as seguintes informações sobre o entrevistado: dados pessoais, clínicos, socioeconômicos, dietéticos, estilo de vida (fumo e álcool), prática de atividade física e presença de história familiar para doenças cardiovasculares. O questionário e o Termo de Consentimento Livre e Esclarecido (TCLE) foram enviados para os pais, junto com uma circular, e só participaram da pesquisa aqueles que devolveram os documentos completamente preenchidos e assinados. Dos 580 participantes convidados, nove não entregaram o TCLE assinado, por isso não puderam fazer parte do estudo. O TCLE e o protocolo de pesquisa foram aprovados pelo Comitê de Ética do Hospital Universitário João de Barros Barreto (2228/05).

Os seguintes fatores de risco foram investigados neste estudo: obesidade, hipertensão arterial, dislipidemia, diabetes, tabagismo, sedentarismo e dieta aterogênica. Suas distribuições foram analisadas segundo gênero, grupo etário e classe econômica.

Hipertensão arterial sistêmica foi considerada como a média (de duas medidas) da pressão arterial sistólica (PAS) e/ou diastólica (PAD) no percentil igual a ou acima de 95, e pressão normal alta (pré-hipertensão), quando a PAS e/ou a PAD situavam-se entre os percentis 90 e 95, ambas ajustadas para o percentil de altura relativo a idade e sexo. Todas as medidas foram realizadas por um mesmo enfermeiro da equipe, seguindo-se as recomendações metodológicas do III Consenso Brasileiro de Hipertensão Arterial e do Update on the 1987 Task Force Report on High 
Blood Pressure in Children and Adolescents, resumidas por Koch 10.

As variáveis relativas aos hábitos de vida foram: tabagismo (fumante; nunca fumou ou exfumante); frequência de uso de bebida alcoólica (abstinente; bebedor ocasional - até três vezes por mês; consumo de risco - acima de duas vezes por semana) e atividade física (qualquer atividade realizada de forma regular, em contexto escolar e extraescolar), aeróbica ou anaeróbica, a partir de 150 minutos por semana. Abaixo desse tempo, o escolar foi classificado como sedentário, não sendo computado o tempo de deslocamento para escola.

A qualidade da dieta foi avaliada mediante escores. O escore alimentar foi adaptado do estudo de Chiara \& Sichieri 11, cuja base foi um questionário de frequência alimentar simplificado (QFAS). Em cada grupo de alimentos do QFAS, foi atribuída uma pontuação em relação à frequência de consumo. Considerando o risco cardiovascular, o consumo foi classificado como elevado (entre 101 e 119 pontos) e excessivo ou aterogênico (igual a ou maior que 120 pontos). A classificação de dislipidemia foi atribuída ao escolar que apresentou qualquer alteração laboratorial, isolada ou combinada, do perfil lipídico - colesterol total (CT), LDL-colesterol (LDL-c), HDL-colesterol (HDL-c) e triglicerídeos (TG).

Para a classificação socioeconômica das famílias dos estudantes, utilizou-se o critério da Associação Brasileira de Empresas de Pesquisa 12, e a renda familiar foi determinada com base no salário mínimo vigente na época $(\mathrm{R} \$ 300,00)$.

\section{Antropometria}

A massa corporal foi medida utilizando-se uma balança digital eletrônica com capacidade de até 150 kg, com precisão de 50 g, (marca Plenna, São Paulo, Brasil). A estatura foi medida com auxílio de uma fita métrica inextensível de 1,5 m fixada perpendicularmente em uma parede plana, após $1 \mathrm{~m}$ do chão, sem rodapé. As mensurações foram realizadas em duplicata, utilizando-se, depois, a média dos valores obtidos, com o objetivo de minimizar possíveis erros de medição.

Para análise, o índice de massa corporal (IMC= $\mathrm{kg} / \mathrm{m}^{2}$ ) foi ajustado para idade e sexo de cada participante, baseando-se nos gráficos de crescimento da Organização Mundial da Saúde (OMS) de 2007 13. Os valores obtidos de IMC foram interpretados em valores de Z score para melhor aplicação dos testes estatísticos. Participantes foram considerados com sobrepeso se o escore $\mathrm{z}$ estivesse entre $>+1 \leq+2$ (correspondente $\geq$ percentil 85 e $\leq$ percentil 97) e obesos se o escore $\mathrm{Z}$ obtido fosse $>+2$ (correspondente $>$ percentil
97). Os escolares classificados pelo IMC acima de $+1 \mathrm{Z}$ foram reagrupados como com excesso de peso, e aqueles abaixo de $+1 \mathrm{Z}$, como sem excesso de peso.

A classificação do status de peso obedeceu ao critério do Ministério da Saúde: escolares menores de dez anos foram denominados crianças e escolares a partir de dez anos, adolescentes.

\section{Perfil bioquímico}

A coleta de sangue foi realizada na própria escola, após 12 horas de jejum feito por cada participante, a fim de se determinar o perfil lipoproteico sérico e da glicemia. No mesmo dia, as amostras foram processadas, e o soro foi analisado em equipamento semiautomatizado (espectrofotômetro SP 2000 UV, marca: Spectrum Laboratories, Rancho Domínguez, Estados Unidos). A análise foi realizada no Laboratório de Erros Inatos do Metabolismo (LEIM) da Universidade Federal do Pará (UFPA). O CT sanguíneo, os TG e o HDL-c foram determinados por métodos enzimáticos, utilizando-se os reagentes da Labtest (Lagoa Santa, Brasil). As concentrações plasmáticas de glicose em jejum foram determinadas pelo método da oxidase (Konelab 60i, Wiener Lab, Rosario, Argentina). O LDL-c foi calculado por meio da equação: LDL-colesterol = colesterol total - (HDL-colesterol + triglicerídeo/5). Os limites de corte utilizados para a avaliação dos limites séricos do CT, TG e HDL-c seguiram a orientação da National Cholesterol Education Program (NCEP) 4; para a glicemia de jejum, o parâmetro foi o da American Diabetes Association 14. Foram considerados níveis indesejáveis de CT e LDL-c valores acima de $170 \mathrm{mg} / \mathrm{dL}$ e $110 \mathrm{mg} / \mathrm{dL}$ e elevados acima de $200 \mathrm{mg} / \mathrm{dL}$ e $130 \mathrm{mg} / \mathrm{dL}$, respectivamente. O termo dislipidemia foi empregado quando se observou qualquer alteração do perfil lipídico (lipoproteínas), isolada ou combinada.

\section{Análise estatística}

Os dados foram analisados com auxílio do programa Epi Info, versão 6.04b (Centers for Disease Control and Prevention, Atlanta, Estados Unidos), por meio do qual também foram calculadas as frequências absolutas e relativas (proporções) dos fatores de risco do estudo quanto ao gênero, idade e nível socioeconômico. Para testar a diferença entre proporções, foi utilizado o teste qui-quadrado ou o teste exato de Fisher, quando necessário.

Após a verificação da consistência dos dados, foram realizadas as análises utilizando-se o pacote estatístico IBM SPSS, versão 19.0 (IBM Corp., Armonk, Estados Unidos). Odds ratio (OR) e 
intervalos de 95\% de confiança (IC95\%) também foram calculados. A regressão binária logística foi realizada incluindo-se as variáveis que apresentaram valor de $\mathrm{p}<0,05$ no teste do qui-quadrado. Para investigar a associação entre o estilo de vida e o perfil lipídico, a OR foi ajustada para gênero e idade, sendo determinada por regressão logística binária. Todas as análises consideraram o efeito do delineamento do estudo e foi adotado um nível de significância de $5 \%$.

\section{Resultados}

Dos 571 sujeitos selecionados, 14 foram excluídos do estudo por desistirem da coleta de sangue, totalizando uma amostra final de 557 escolares (437 da rede pública e 120 da rede particular). A distribuição das características sociodemográficas da amostra está descrita na Tabela 1; a prevalência dos fatores de risco investigados no estudo está apresentada na Tabela 2.

Do total dos escolares, $20,4 \%$ apresentaram excesso de peso; $2,7 \%$, colesterol total acima de $200 \mathrm{mg} / \mathrm{dL} ; 3,6 \%$, LDL-c acima de $130 \mathrm{mg} / \mathrm{dL}$; e $26,9 \%$ HDL baixo $(<40 \mathrm{mg} / \mathrm{dL}$ para as crianças e $35 \mathrm{mg} / \mathrm{dL}$ para os adolescentes). Ao se analisar a Tabela 1, observou-se que mais da metade dos estudantes eram adolescentes $(75,4 \%)$, pertenciam à classe econômica baixa $(82,1 \%)$, suas mães tinham, no mínimo, nove anos de estudo (65,5\%) e eram, em minoria, negros $(10,4 \%)$.

Excesso de peso, dislipidemia, história familiar positiva para doenças cardiovasculares e sedentarismo foram os fatores de risco com maior prevalência dentre os investigados. Aproximadamente $39 \%$ das crianças analisadas apresentaram três ou mais fatores de risco agrupados. Este grupo etário tendeu a apresentar maior número de casos de indivíduos obesos, sedentários e dislipidêmicos em comparação aos escolares adolescentes, exceto no que diz respeito ao fumo (Tabela 2). Os adolescentes na faixa de 13 a 16 anos foram os que apresentaram menor chance de desenvolver excesso de peso, hipercolesterolemia (LDL elevado) e hipertrigliceredemia em comparação ao restante da amostra.

Em relação ao gênero, foi constatado que os escolares do sexo masculino se apresentaram mais ativos e menos propensos a desenvolver pré-hipertensão em comparação com os do sexo feminino (Tabelas 2 e 3). A prevalência de obesidade foi menor entre os escolares de maior faixa etária $(\mathrm{p}<0,001)$ e nas classes econômicas mais baixas $(\mathrm{p}<0,07)$.

Por meio da análise bivariada, foi possível confirmar que os escolares menores de dez anos apresentaram maior chance de desenvol-
Tabela 1

Características sociodemográficas da população em estudo. Belém, Pará, Brasil.

\begin{tabular}{|c|c|c|}
\hline Variáveis & $\mathbf{n}$ & $\%$ \\
\hline \multicolumn{3}{|l|}{ Sexo } \\
\hline Feminino & 294 & 52,8 \\
\hline Masculino & 263 & 47,2 \\
\hline \multicolumn{3}{|l|}{ Idade (anos) } \\
\hline $6 \vdash 10$ & 137 & 24,6 \\
\hline $10 \vdash 13$ & 141 & 25,3 \\
\hline $13 \vdash 16$ & 131 & 23,5 \\
\hline $16 \vdash 20$ & 148 & 26,6 \\
\hline \multicolumn{3}{|l|}{ Raça } \\
\hline Branco & 267 & 47,9 \\
\hline Pardo & 232 & 41,7 \\
\hline Negro & 58 & 10,4 \\
\hline \multicolumn{3}{|c|}{ Tipo de escola } \\
\hline Pública & 437 & 78,5 \\
\hline Privada & 120 & 21,5 \\
\hline \multicolumn{3}{|c|}{ Escolaridade materna (anos) } \\
\hline$\leq 8$ & 191 & 34,5 \\
\hline $9-12$ & 262 & 47,4 \\
\hline$\geq 13$ & 100 & 18,1 \\
\hline \multicolumn{3}{|c|}{ Classe econômica * } \\
\hline$A$ e $B$ & 99 & 17,9 \\
\hline C & 297 & 53,8 \\
\hline$D$ e $E$ & 156 & 28,3 \\
\hline
\end{tabular}

* Cinco não informaram a renda familiar.

ver excesso de peso e alterações do perfil lipídico (LDL-c e TG) na amostra (Tabela 3). As crianças também apresentaram maior chance de desenvolver HDL-c baixo em comparação com os escolares adolescentes $(\mathrm{OR}=2,3$; IC95\%: 1,5-3,5; $\mathrm{p}<0,001$ ). As variáveis independentes, como o poder aquisitivo e a escolaridade materna, só demonstraram associação positiva e significativa entre os escolares que apresentaram IMC acima do percentil 85.

A análise de regressão binária logística ajustada para idade e sexo demonstrou que os escolares com excesso de peso foram os indivíduos da amostra mais propensos a desenvolver dislipidemia, principalmente hipertrigliceredemia Quanto ao estilo de vida, o sedentarismo e a dieta inadequada apresentaram associação significativa apenas com um dos componentes do perfil lipídico investigados. Não foi observada associação entre hipertensão arterial e os outros fatores de risco relacionados ao estilo de vida (Tabela 4). 
Prevalência de alterações antropométricas, clínicas e dietéticas da população em estudo, segundo as variáveis selecionadas. Belém, Pará, Brasil.

\begin{tabular}{|c|c|c|c|c|c|c|c|c|c|c|c|c|}
\hline \multirow[t]{2}{*}{ Fatores de risco } & \multicolumn{2}{|c|}{ Total } & \multicolumn{3}{|c|}{ Gênero (\%) } & \multicolumn{3}{|c|}{ Faixa etária (anos) (\%) } & \multicolumn{4}{|c|}{ Classe econômica (\%) * } \\
\hline & $\mathrm{n}$ & $\%$ & $\begin{array}{c}M \\
(n=294)\end{array}$ & $\begin{array}{c}F \\
(n=263)\end{array}$ & $\begin{array}{l}\text { Valor } \\
\text { de } p\end{array}$ & $\begin{array}{c}<10 \\
(n=137)\end{array}$ & $\begin{array}{c}>10 \\
(n=420)\end{array}$ & $\begin{array}{l}\text { Valor } \\
\text { de } p\end{array}$ & $\begin{array}{c}A+B \\
(n=99)\end{array}$ & $\begin{array}{c}C \\
(n=297)\end{array}$ & $\begin{array}{c}D+E \\
(n=156)\end{array}$ & $\begin{array}{l}\text { Valor } \\
\text { de } p\end{array}$ \\
\hline Sobrepeso & 64 & 11,5 & 10,3 & 12,6 & 0,19 & 12,4 & 11,2 & 0,35 & 16,2 & 9,1 & 7,7 & 0,19 \\
\hline Obesidade & 55 & 8,9 & 12,2 & 7,8 & 0,05 & 19,0 & 6,9 & $<0,01$ & 12,1 & 13,5 & 7,7 & 0,07 \\
\hline Pré-hipertensão & 208 & 37,3 & 30,4 & 43,5 & 0,01 & 40,6 & 37,0 & 0,60 & 36,7 & 37,4 & 39,5 & 0,87 \\
\hline Hipertensão & 40 & 7,2 & 8,0 & 6,5 & 0,01 & 8,3 & 7,0 & 0,32 & 5,1 & 7,5 & 7,9 & 0,69 \\
\hline $\begin{array}{l}\text { História familiar para } \\
\text { doença cardiovascular }\end{array}$ & 162 & 29,1 & 27,0 & 31,0 & 0,37 & 29,2 & 29,0 & 0,85 & 32,3 & 29,6 & 25,6 & 0,19 \\
\hline Glicemia alta & 10 & 1,8 & 2,3 & 1,4 & 0,41 & 2,2 & 1,7 & 0,71 & 2,0 & 2,0 & 1,3 & 0,83 \\
\hline Sedentarismo & 369 & 66,2 & 46,0 & 77,6 & $<0,01$ & 73,7 & 64,0 & 0,04 & 62,2 & 65,0 & 71,2 & 0,27 \\
\hline $\begin{array}{l}\text { Álcool (consumo de } \\
\text { risco) }\end{array}$ & 5 & 0,9 & 1,5 & 0,3 & 0,14 & 0,0 & 1,2 & 0,20 & 0,0 & 1,3 & 1,0 & 0,35 \\
\hline Fumo & 4 & 0,7 & 0,4 & 1,0 & 0,70 & 0,0 & 1,0 & 0,13 & 0,0 & 1,3 & 0,0 & 0,18 \\
\hline Dislipidemia ** & 268 & 48,1 & 49,0 & 47,3 & 0,68 & 62,0 & 43,6 & $<0,01$ & 41,4 & 50,5 & 46,8 & 0,28 \\
\hline $\mathrm{LDL}_{-\mathrm{c}}{ }^{* \star *} \geq 110 \mathrm{mg} / \mathrm{dL}$ & 88 & 15,8 & 16,0 & 15,6 & 0,92 & 19,7 & 14,5 & 0,15 & 19,2 & 15,2 & 14,7 & 0,55 \\
\hline HDL baixo \# & 150 & 26,9 & 29,7 & 24,5 & 0,17 & 40,1 & 22,6 & $<0,01$ & 21,2 & 28,6 & 27,6 & 0,35 \\
\hline Triglicerídeo alto \#\# & 94 & 16,9 & 14,8 & 18,7 & 0,17 & 23,4 & 14,8 & 0,02 & 14,1 & 17,5 & 17,9 & 0,70 \\
\hline Dieta aterogênica & 24 & 4,3 & 6,5 & 9,5 & 0,19 & 9,5 & 7,6 & 0,49 & 6,1 & 7,7 & 10,3 & 0,46 \\
\hline
\end{tabular}

* Três não informaram;

** Qualquer alteração do perfil lipídico;

*** Lipoproteína baixa densidade;

\# Lipoproteína de alta densidade (baixo: $<40 \mathrm{mg} / \mathrm{dL}$ para crianças e $<35 \mathrm{mg} / \mathrm{dL}$ para adolescentes);

\#\# Elevado $>100 \mathrm{mg} / \mathrm{dL}$ para crianças e $>130 \mathrm{mg} / \mathrm{dL}$ para adolescentes.

Escolares sedentários apresentaram mais chance de desenvolver excesso de peso do que os ativos $(\mathrm{OR}=1,94 ;$ IC95\%: 1,8-3,2; $\mathrm{p}=0,009)$.

\section{Discussão}

O presente estudo representa uma importante contribuição de dados epidemiológicos para a Região Norte, relevantes no cenário científico nacional e internacional, principalmente em razão da ausência, na literatura, de trabalhos que investiguem fatores de risco para doenças cardiovasculares em uma amostra representativa de escolares dessa região. O número de fatores de risco analisados também merece destaque, pois foi superior à média apresentada na literatura para a faixa etária pediátrica 15,16.

Os resultados desta investigação indicaram que os percentuais de participantes obesos, hipertensos, dislipidêmicos e sedentários foram altos na região e que as crianças $(<10$ anos) apresentaram o maior número de fatores de risco agrupados. Apesar de a prevalência de excesso de peso $(20,4 \%)$ encontrada ser inferior à verificada em alguns estudos recentes realizados na Região
Sudeste 15,17 , ela foi superior à encontrada na maioria de outros estudos realizados no país 11,16 e no mundo 18 nos últimos dez anos. Além disso, nessa população, foi observada associação entre obesidade e alterações do perfil lipídico, principalmente entre os escolares sedentários e menores de dez anos, ratificando a importância do estímulo à alimentação saudável e à prática física regular desde a fase pré-escolar 5 .

É possível que os achados deste estudo estejam mais estritamente relacionados com o percentual de sedentarismo encontrado do que com a qualidade da alimentação consumida na faixa etária investigada. Vale destacar que, embora a oferta de salgadinhos, biscoitos (snacks) e sanduíches à base de fritura e o baixo consumo de frutas e legumes 19 continuem sendo práticas vivenciadas nas cantinas dos colégios, o percentual de estudantes que consumiram dieta aterogênica foi pequeno. Cabe esclarecer também que não se descarta a possibilidade de existência de relação entre a quantidade de calorias e de sódio consumidos pelos escolares e os achados percentuais de hipertensão e excesso de peso. Tais dados não foram foco de investigação nesta pesquisa. 
Tabela 3

Análise bivariada entre fatores de risco para doenças cardiovasculares e as variáveis sociodemográficas da população em estudo. Belém, Pará, Brasil.

\begin{tabular}{|c|c|c|c|c|c|c|c|c|c|c|c|c|c|c|c|}
\hline \multirow{2}{*}{$\begin{array}{l}\text { Fatores de } \\
\text { risco }\end{array}$} & \multicolumn{3}{|c|}{ IMC > p85 } & \multicolumn{3}{|c|}{$\mathrm{PA}>\mathrm{p} 90$} & \multicolumn{3}{|c|}{$\mathrm{LDL}-\mathrm{c} \geq 110 \mathrm{mg} / \mathrm{dL}$} & \multicolumn{3}{|c|}{ TG elevado * } & \multicolumn{3}{|c|}{ Sedentarismo ** } \\
\hline & OR & IC95\% & $\begin{array}{l}\text { Valor } \\
\text { de } p\end{array}$ & OR & IC95\% & $\begin{array}{l}\text { Valor } \\
\text { de } p\end{array}$ & OR & IC95\% & $\begin{array}{l}\text { Valor } \\
\text { de } p\end{array}$ & OR & IC95\% & $\begin{array}{l}\text { Valor } \\
\text { de } p\end{array}$ & OR & IC95\% & $\begin{array}{l}\text { Valor } \\
\text { de } p\end{array}$ \\
\hline \multicolumn{16}{|l|}{ Sexo } \\
\hline Masculino & 1,00 & Referência & & 1,00 & Referência & & 1,00 & Referência & & 1,00 & Referência & & 1,00 & Referência & \\
\hline Feminino & 0,89 & $0,59-1,33$ & 0,56 & 1,63 & $1,16-2,29$ & 0,01 & 0,99 & $0,60-1,60$ & 0,95 & 1,32 & $0,84-2,07$ & 0,22 & 2,97 & $2,06-4,27$ & 0.00 \\
\hline \multicolumn{16}{|l|}{$\begin{array}{l}\text { Grupo etário } \\
\text { (anos) }\end{array}$} \\
\hline $16 \vdash 20$ & 1,00 & Referência & & 1,00 & Referência & & 1,00 & Referência & & 1,00 & Referência & & 1,00 & Referência & \\
\hline $13 \vdash 16$ & 1,93 & $0,94-3,95$ & 0,07 & 1,29 & $0,56-1,51$ & 0,72 & 0,91 & $0,42-2,00$ & 0,82 & 0,61 & $0,27-1,35$ & 0,22 & 1,24 & $0,74-2,10$ & 0,41 \\
\hline $10 \vdash 13$ & 3,79 & $2,10-8,67$ & $<0,01$ & 1,22 & $0,76-2,00$ & 0,40 & 2,10 & $1,06-4,07$ & 0,03 & 1,85 & $0,97-3,53$ & 0,06 & 1,68 & $0,90-2,82$ & 0,05 \\
\hline $6 \vdash 10$ & 4,38 & $2,40-10,0$ & $<0,01$ & 00,91 & $0,78-2,14$ & 0,41 & 2,15 & $1,07-4,30$ & 0.00 & 1,95 & $1,01-3,75$ & 0,04 & 1,86 & $1,13-3,08$ & 0,02 \\
\hline \multirow{2}{*}{\multicolumn{16}{|c|}{$\begin{array}{l}\text { Escolaridade } \\
\text { da mãe (anos) }\end{array}$}} \\
\hline & & & & & & & & & & & & & & & \\
\hline$\leq 8$ & 1,00 & Referência & & 1,00 & Referência & & 1,00 & Referência & & 1,00 & Referência & & 1,00 & Referência & \\
\hline $9-12$ & 1,6 & $0,99-2,6$ & 0,06 & 0,94 & $0,64-1,37$ & 0,74 & 0,53 & $0,51-1,42$ & 0,53 & 1,22 & $0,73-2,04$ & 0,45 & 1,38 & $0,93-2,06$ & 0,11 \\
\hline$\geq 13$ & 2,3 & $1,27-4,09$ & 0,01 & 1,1 & $0,67-1,78$ & 0,72 & 0,87 & $0,49-1,82$ & 0,87 & 1,46 & $0,77-2,74$ & 0,25 & 0,96 & $0,56-1,63$ & 0,90 \\
\hline \multicolumn{16}{|l|}{ Classe } \\
\hline \multicolumn{16}{|l|}{ econômica } \\
\hline$D$ e $E$ & 1,00 & Referência & & 1,00 & Referência & & 1,00 & Referência & & 1,00 & Referência & & 1,00 & Referência & \\
\hline C & 1,60 & $0,96-2,68$ & 0,09 & 1,36 & $0,77-2,39$ & 0,29 & 1,03 & $0,60-1,78$ & 0,98 & 1,17 & $0,54-2,54$ & 0,69 & 1,35 & $0,87-2,02$ & 0,19 \\
\hline$A$ e $B$ & 2,17 & $1,17-4,02$ & 0,02 & 1,19 & $0,73-1,94$ & 0,59 & 1,37 & $0,70-2,68$ & 0,45 & 1,29 & $0,66-2,53$ & 0,46 & 1,50 & $0,88-2,56$ & 0,14 \\
\hline
\end{tabular}

IMC: índice de massa corporal; LDL-c: lipoproteína de baixa densidade; PA: pressão arterial; OR: odds ratio; TG: triglicerídeo.

* Elevado $>100 \mathrm{mg} / \mathrm{dL}$ para crianças e $>130 \mathrm{mg} / \mathrm{dL}$ para adolescentes;

** Atividade física $<150$ minutos/semana.

Tabela 4

Odds ratio (OR) ajustada entre fatores de risco e estilo de vida da população em estudo. Belém, Pará, Brasil.

\begin{tabular}{|c|c|c|c|c|c|c|c|c|c|c|c|c|c|c|c|}
\hline & \multicolumn{3}{|c|}{$\mathrm{CT}>170 \mathrm{mg} / \mathrm{dL}$} & \multicolumn{3}{|c|}{ LDL-c $\geq 110 \mathrm{mg} / \mathrm{dL}$} & \multicolumn{3}{|c|}{ TG elevado * } & \multicolumn{3}{|c|}{ HDL-c baixo ** } & \multicolumn{3}{|c|}{$\mathrm{PA}>\mathrm{p} 90$} \\
\hline & $\begin{array}{l}\text { OR } \\
\star \star \star\end{array}$ & IC95\% & $\begin{array}{l}\text { Valor } \\
\text { de } p\end{array}$ & OR & IC95\% & $\begin{array}{l}\text { Valor } \\
\text { de } p\end{array}$ & OR & IC95\% & $\begin{array}{l}\text { Valor } \\
\text { de } p\end{array}$ & OR & IC95\% & $\begin{array}{l}\text { Valor } \\
\text { de } p\end{array}$ & OR & IC95\% & $\begin{array}{l}\text { Valor } \\
\text { de } p\end{array}$ \\
\hline \multicolumn{16}{|l|}{ Excesso de } \\
\hline \multicolumn{16}{|l|}{ peso } \\
\hline Não & 1,00 & Referência & & 1,00 & Referência & & 1,00 & Referência & & 1,00 & Referência & & 1,00 & Referência & \\
\hline Sim & 1,73 & $1.08-2,77$ & 0,02 & 1,85 & $1,10-3,10$ & 0,02 & 3,20 & $1,99-5,15$ & $<0,01$ & 0,64 & $0,41-1,05$ & 0,05 & 1,20 & $0,80-1,82$ & 0.43 \\
\hline \multicolumn{16}{|l|}{ Sedentarismo } \\
\hline Não & 1,00 & Referência & & 1,00 & Referência & & 1,00 & Referência & & 1,00 & Referência & & 1,00 & Referência & \\
\hline Sim & 1,22 & $0,32-1,81$ & 0,32 & 11,34 & $0,82-2,20$ & 0,25 & 1,52 & $0,89-2,60$ & 0,04 & 1,04 & $0,68-1,59$ & 0,84 & 1,25 & $0,90-1,80$ & 0.24 \\
\hline \multicolumn{16}{|l|}{ Dieta } \\
\hline $\begin{array}{l}\text { Não } \\
\text { aterogênica }\end{array}$ & 1,00 & Referência & & 1,00 & Referência & & 1,00 & Referência & & 1,00 & Referência & & 1,00 & Referência & \\
\hline Aterogênica & 2,32 & $1,09-4,94$ & 0,03 & 0,65 & $0,25-1,69$ & 0,49 & 1,91 & $0,95-3,80$ & 0,07 & 0,56 & $0,29-1,09$ & 0,09 & 1,00 & $0,54-1,85$ & 0,99 \\
\hline
\end{tabular}

CT: colesterol total; HDL-c: lipoproteína de alta densidade; LDL-c: lipoproteína baixa densidade; PA: pressão arterial; TG: triglicerídeo.

* Elevado $>100 \mathrm{mg} / \mathrm{dL}$ para crianças e $>130 \mathrm{mg} / \mathrm{dL}$ para adolescentes;

** Baixo $<40 \mathrm{mg} / \mathrm{dL}$ para crianças e $<35 \mathrm{mg} / \mathrm{dL}$ para adolescentes;

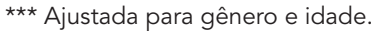


Quanto à atividade física, mesmo sendo considerada uma das importantes medidas para prevenção do desenvolvimento de obesidade e da doença coronariana, trata-se de prática ainda pouco vivenciada na fase escolar, principalmente no sexo feminino 20,21. Em crianças, os benefícios associados à atividade física incluem controle do peso, efeitos atenuadores sobre as concentrações de colesterol e a resistência insulínica, pressão arterial baixa, bem-estar e maior predisposição para vida ativa na adolescência 5,22 .

As principais barreiras relatadas que impedem o engajamento em atividade física durante a infância devem-se à ascensão do lazer passivo (televisão e video game) e ao declínio do lazer ativo (pular corda, elástico e andar de bicicleta); na adolescência, por sua vez, o impedimento pode advir de fatores ambientais e socioculturais 21,23. Nesse contexto, observa-se que, desde cedo, adolescentes do sexo feminino são direcionadas para o cuidado com a família e afazeres domésticos, enquanto os do sexo masculino são orientados para atividades laborais e de intensidade mais vigorosa 24 .

Em relação ao perfil lipídico, constatou-se que os percentuais de dislipidemia (CT e LDL) encontrados foram bem inferiores aos de estudos realizados em outras regiões do país 2,25 que seguiram o critério da NCEP como ponto de corte $(\mathrm{CT}>200 \mathrm{mg} / \mathrm{dL})$. Porém, a prevalência de $15,8 \%$ de hipercolesterolemia encontrada na cidade de Belém foi cerca de $60 \%$ maior do que a observada no único estudo similar realizado na mesma região (10,9\%), mas não publicado, o qual adotou como ponto de corte CT > $170 \mathrm{mg} / \mathrm{dL}$ 9. Quanto à HDL-c, os=percentuais de inadequação encontrados, principalmente nas crianças, corroboraram outros estudos brasileiros 26,27. Embora, do ponto de vista terapêutico, a redução do LDL-c seja ainda o principal foco na terapia hipolipemiante para a prevenção e tratamento da doença arterial coronariana, há provas contundentes de que baixo nível de plasma de HDL-c é um importante fator de risco independente para doenças cardiovasculares 27. Ademais, nossos resultados demonstraram associação positiva entre os níveis elevados de TG e dois importantes fatores de risco para doenças cardiovasculares: obesidade e sedentarismo, principalmente entre as crianças $(<10$ anos). Este achado foi concordante com os de outros estudos brasileiros 3,28,29. Altas concentrações de TG contribuem para o seu depósito na parede do vaso e o início do processo de acumulação do LDL-c, que está fortemente associado ao risco de desenvolvimento da aterosclerose 5 . Apesar disso, a hipertrigliceridemia é o distúrbio lipídico de mais fácil monitoração, podendo ser controlado satisfatoriamente por mudanças do estilo de vida.

Com base nos dados apresentados, destacase a importância de se diagnosticar precocemente a dislipidemia nos primeiros ciclos da vida. No entanto, a comparação dos resultados com os de outras regiões do país e do mundo é tarefa difícil, uma vez que, na literatura, existem diferentes pontos de corte para a classificação dessa disfunção.

No que se refere à hipertensão, vários estudos têm demonstrado que o excesso de peso está positivamente correlacionado com a ocorrência de pré-hipertensão em crianças e adolescentes, associação que aumenta o risco de seu desenvolvimento no adulto ${ }^{30}$. Mesmo que a prevalência de hipertensão encontrada entre os escolares tenha sido expressiva, não foi observada associação com outros fatores de riscos investigados neste estudo. A diferença encontrada entre os sexos talvez possa ser explicada pelo percentual de inatividade física registrado no sexo feminino e pela ação dos hormônios sexuais nesse gênero.

Outro aspecto relevante foi a análise dos fatores de risco segundo o poder aquisitivo e a escolaridade materna. Constatou-se que os escolares provenientes de famílias de maior poder aquisitivo (A e B) e escolaridade materna ( $\geq 13$ anos) apresentaram duas vezes mais chance de desenvolver excesso de peso do que os dos outros estratos, apesar de essa associação não ter sido observada entre os demais fatores de risco avaliados no presente estudo. Esse achado pode ser justificado pelo fato de que a prevalência de sobrepeso e a obesidade sofrem interferência do nível socioeconômico e da escolaridade, na medida em que estes determinam maior acesso aos alimentos de alta densidade calórica, entre outras condições 31 .

Diferentes estudos também mostram que as mulheres com maior escolaridade tendem a se inserir no mercado de trabalho e a realizar grande parte de sua alimentação no trabalho. Segundo a Pesquisa de Orçamentos Familiares 32, refeições fora do domicílio são, geralmente, constituídas de alimentos industrializados e ultraprocessados, de alto valor calórico, ricos em gordura saturada e sódio. Esse padrão alimentar é, muitas vezes, repetido dentro do domicílio, contribuindo para o desenvolvimento do quadro de obesidade para toda a família. Em adição, filhos de mães que trabalham fora tendem, comumente, a apresentar maior tempo de lazer passivo (televisão, video game e computador) do que filhos de mães que não trabalham, contribuindo para isso o quadro atual de violência urbana, que restringe cada vez mais as atividades fora do domicílio (futebol, piques ou corridas) 33 . 
Outros fatores de risco investigados, como fumo, álcool, diabetes e dieta inadequada, apresentaram número pequeno de casos e não diferiram estatisticamente entre os gêneros, grupos etários e diferentes classes econômicas. Apesar do expressivo percentual de história familiar positiva para doenças cardiovasculares $(24,1 \%=$ 134), encontrado no estudo, essa variável é difícil de ser analisada de forma isolada por sofrer influência de outros fatores, como os culturais e genéticos.

Em face dos resultados, todos os familiares dos escolares que apresentaram fatores de risco modificáveis foram notificados e orientados pelo pesquisador a levarem os jovens a um serviço de saúde, prevenindo, assim, complicações cardiovasculares na vida adulta.

Os achados reforçam a importância de se ampliar a investigação dos fatores de risco e fatores associados na população pediátrica, principalmente em regiões brasileiras que ainda carecem de estudos; é necessário, também, padronizarem-se os critérios utilizados para a classificação desses fatores a fim de facilitar comparações locais e/ou regionais e permitir a construção de um panorama geral dos principais fatores de risco distribuídos no país. Além disso, desde a primeira infância, devem ser implantadas estratégias para controle de excesso de peso, por meio de uma alimentação balanceada e da prática física regular, para que se possa reduzir de forma efetiva a prevalência de fatores de risco em escolares de Belém. Tais ações devem ser constantemente estimuladas no espaço escolar, na família e na comunidade, para que possam realmente atenuar o desenvolvimento, na vida adulta, de doenças crônicas não transmissíveis.

\section{Resumen}

Este estudio transversal tuvo por objetivo identificar los factores de riesgo para enfermedades cardiovasculares en una muestra de 557 escolares (6-19 años) de Belém, Pará, Brasil, estratificada y por conglomerados. Los factores de riesgo investigados fueron: la obesidad, la hipertensión, la dislipidemia, la diabetes, el tabaquismo, la inactividad física y dieta aterogénica. Las variables sociodemográficas y relacionadas con el estilo de vida se probaron con el modelo de regresión logística binaria. Los factores de riesgo más prevalentes fueron: sobrepeso (20,4\%), dislipidemia (48,1\%) e inactividad física (66,2\%). Se descubrió que los estudiantes menores de
10 años, provenientes de familias con mayores ingresos y mayor educación de la madre eran más propensos a desarrollar sobrepeso y son más propensos a desarrollar hipercolesterolemia y hipertrigliceredemia. En este contexto, es necesario, incluso en la infancia, la implementación de estrategias para controlar el exceso de peso mediante una dieta equilibrada y ejercicio físico regular, puesto que puede reducir eficazmente la preva lencia de factores de riesgo en escolares de esta ciudad.

Estudiantes; Enfermedades Cardiovasculares; Factores de Riesgo 


\section{Colaboradores}

S. A. Ribas elaborou o projeto inicial, executou-o, analisou os resultados, redigiu e revisou o artigo. L. C. Santana da Silva participou da elaboração do projeto inicial, analisou os resultados e revisou o artigo.

\section{Agradecimentos}

Agradecemos aos diretores dos colégios e aos familiares dos estudantes, pela oportunidade de desenvolver este trabalho. À Luana Monteiro e ao Alexandre de Mota Pinto, pelas críticas e sugestões tão enriquecedoras.

\section{Referências}

1. Rede Interagencial de Informações para a Saúde Indicadores básicos para saúde no Brasil: conceitos e aplicações. 2a Ed. Brasília: Organização PanAmericana da Saúde; 2008.

2. Coronelli CLS, Moura EC. Hipercolesterolemia em escolares e seus fatores de risco. Rev Saúde Pública 2003; 37:24-31.

3. Ribas SA, Santana da Silva LC. Anthropometric indices: predictors of dyslipidemia in children and adolescents from north of Brazil. Nutr Hosp 2012; 27:1212-9.

4. National Cholesterol Education Program. Highlights of the report of the expert panel on blood cholesterol levels in children and adolescents. Pediatrics 1992; 89:495-501

5. Rodrigues AN, Abreu GR, Resende RS, Gonçalves WLS, Gouvea AS. Cardiovascular risk factor investigation: a pediatric issue. Int J Gen Med 2013, 6:57-66.

6. Kannel WB. Risk stratification in hypertension: new insights from the Framingham Study. Am J Hypertens 2000; 13(1 pt 2):3S-10S.
7. Schmitz BAS, Recine E, Cardoso GT, Silva JRM, Amorim NFA, Bernardon R, et al. A escola promovendo hábitos alimentares saudáveis: uma proposta metodológica de capacitação para educadores e donos de cantina escolar. Cad Saúde Pública 2008; 24 Suppl 2:S312-22.

8. Instituto Nacional de Estudos e Pesquisas Educacionais. Censo educacional. Belém: Instituto $\mathrm{Na}-$ cional de Estudos e Pesquisas Educacionais; 2004.

9. Rego SS. Análise dos níveis sanguíneos de colesterol total em estudantes adolescentes de Belém, Pará [Dissertação de Mestrado]. São Paulo: Escola Paulista de Medicina, Universidade Federal de São Paulo; 1999.

10. Koch VH. Pressão arterial em pediatria: aspectos metodológicos e normatização. Rev Bras Hipertens 2000; 7:71-8.

11. Chiara VL, Sichieri R. Consumo alimentar em adolescentes. Questionário simplificado para avaliação de risco cardiovascular. Arq Bras Cardiol 2001; 77:332-6. 
12. Associação Brasileira de Empresas de Pesquisa Critério de classificação econômica Brasil (CCEB). http://www.abep.org/novo/Content.aspx?Con tentID=301 (acessado em 15/Mar/2011).

13. WHO Multicentre Growth Reference Study Group. WHO child growth standards: length/height-forage, weight-for-age,weight-for-length, weight-forheight and body mass index-for-age: methods and development. Geneva: World Health Organization; 2007.

14. American Diabetes Association Position Statement. Diagnosis and classification of diabetes mellitus. Diab Care 2010; 33 Suppl 1:S62-9.

15. Molina MCB, Faria CP, Montero MP, Cade NV, Mill JG. Fatores de risco cardiovascular em crianças de 7 a 10 anos de área urbana, Vitória, Espírito Santo, Brasil. Cad Saúde Pública 2010; 26:909-17.

16. Giussani M, Antolini L, Brambilla P, Pagani M, Zuccotti G, Valsecchi MG, et al. Cardiovascular risk assessment in children: role of physical activity, family history and parental smoking on BMI and blood pressure. J Hypertens 2013; 31:983-92.

17. Pereira A, Guedes AD, Verreschi ITN, Santos RD, Martinez TLR. A obesidade e sua associação com os demais fatores de risco cardiovascular em escolares de Itapetininga, Brasil. Arq Bras Cardiol 2009; 93:253-60.

18. Brotons C, Ribera A, Perich RM, Abrodos D, Magana P, Pablo S, et al. Worldwide distribution of blood lipids and lipoproteins in childhood and adolescence: a review study. Atheroesclerosis 1998; 139:1-9.

19. Perry C, Bishop DB, Taylor GR, Davis M, Story M, Gray C, et al. A randomized school trial of environmental strategies to encourage fruit and vegetable among children. Health Educ Behav 2004; 31: 65-76.

20. Ferreira I, van der Horst K, Wendel-Vos W, Kremers S, van Lenthe FJ, Brug J. Environmental correlates of physical activity in youth - a review and update. Obes Rev 2006; 8:129-54.

21. Fermino RC, Rech CR, Hino AAF, Añez CRR, Reis RS. Atividade física e fatores associados em adolescentes do ensino médio de Curitiba, Brasil. Rev Saúde Pública 2010; 44:986-95.

22. Strong WB, Malina RM, Blimkie CJ, Daniels SR, Dishman RK, Gutin B, et al. Evidence based physical activity for school-age youth. J Pediatr 2005; 146:732-7.
23. Santos MS, Hino AAF, Reis RS, Rodriguez-Añez CR. Prevalência de barreiras para a prática de atividade física em adolescentes. Rev Bras Epidemiol 2010; 13:94-104.

24. Seabra AF, Mendonça DM, Thomis MA, Anjos LA, Maia JA. Determinantes biológicos e sócio-culturais associados à prática de atividade física de adolescentes. Cad Saúde Pública 2008; 24:721-36.

25. Teixeira MA, Veiga GV, Sichieri R. Consumo de gordura e hipercolesterolemia em uma amostra probabilística de estudantes de Niterói, Rio de Janeiro. Arq Bras Endocrinol Metab 2007; 51:65-71.

26. Beck CC, Lopes AS, Giuliano ICB, Borgatto AF. Cardiovacular risk factors in adolescents from a town in the Brazilian South: prevalence and association with sociodemographic variables. Rev Bras Epidemiol 2011; 14:36-49.

27. Seki M, Matsuo T, Carrilho AJF. Prevalence of metabolic syndrome and associated risk factors in Brazilian schoolchildren. Public Health Nutr 2008, 12:947-52.

28. Candido APC, Beneddeto R, Castro APP, Carmo JS, Nicolato RLC, Nascimento-Neto RM, et al. Cardiovascular risk factors in children and adolescents living in an urban area of Southeast of Brazil: Ouro Preto Study. Eur J Pediatr 2009; 168:1373-82.

29. Rinaldi AEM, Oliveira EP, Moreto F, Gabriel GFCP, Corrente JE, Burini RC. Dietary intake and blood lipid profile in overweight and obese schoolchildren. BMC Res Notes 2012; 5:598.

30. Srinivasan SR, Myers L, Berenson GS. Changes in metabolic syndrome variables since childhood in prehypertensive and hypertensive subjects: the Bogalusa Heart Study. Hypertension 2006; 48:33-9.

31. Menezes RCE, Lira PIC, Oliveira JS, Leal VS, Santana SCS, Andrade SLLS, et al. Prevalência e determinantes do excesso de peso em pré-escolares. J Pediatr (Rio J.) 2011; 87:231-7.

32. Instituto Brasileiro de Geografia e Estatística. Pesquisa de orçamentos familiares 2008-2009: análise do consumo alimentar pessoal no Brasil. Rio de Janeiro: Instituto Brasileiro de Geografia e Estatística; 2011

33. Frutuoso MFP, Bovi TG, Gambardella AMD. Adiposidade em adolescentes e obesidade materna. Rev Nutr 2011; 24:5-15.

Recebido em 02/Set/2012

Versão final reapresentada em 31/Jul/2013 Aprovado em 09/Set/2013 